\title{
Measurement of Unique Pupillary Distance using Modified Circle Algorithm
}

\author{
A. F. M. Saifuddin Saif \\ Assistant Professor, Department of Computer Science \\ American International University-Bangladesh \\ Dhaka, Bangladesh \\ Khandaker Tabin Hasan \\ Associate Professor, Department of Computer Science \\ American International University-Bangladesh \\ Dhaka, Bangladesh
}

\author{
Md. Shahadat Hossain \\ M Scholar, Department of Computer Science \\ American International University-Bangladesh \\ Dhaka, Bangladesh \\ Mashiour Rahman \\ Associate Professor, Department of Computer Science \\ American International University-Bangladesh \\ Dhaka, Bangladesh
}

\begin{abstract}
This research investigated several equations and calculated the pupillary distance using pupils center coordinate. The prior research has provided two conceptual frameworks which may give a unique distance to all human. As Pupillary distance provides a core and unique information as like bio-metric for every human. The researcher did not explore the equation or pattern yet. This research proposed a framework called as Pixellary Pupil Distance and analyze the result. This research use pixel to calculate the pupillary distance where subtracts left pupil pixel with the right pupil and sum up with its distance pixel. Finally, the research sum all the pixel subtraction and its distance what this research called pupillary distance. This research builds and describes a custom dataset for test purpose because the pupil is the core element of eyes and this research did not find any precise dataset of it. The result shows the unicity of pupillary distance. This research also shows the computational time, false alarming rate, noise reduction which is described the performance of the purposed framework.
\end{abstract}

\section{Keywords}

Pixellary pupil distance, Pupillary Distance, Bio-metrics, Eyes, Distance Equation, Pupil Detection, Pupil Measurement, Edge Detection

\section{INTRODUCTION}

Pupillary distance is unique. This research has explored the unique measurement of Pupillary Distance. There are many problems which are not defined by the mathematical equation. Researchers are trying to identify mathematical equations which are not defined yet. The unique measurement of Pupillary Distance is one of them. The most popular distance equations of two-point cannot solve the problem. As a result, The research proposed two conceptual frameworks [1] which have been presented distance equation of Pupillary Distance. The research proposed the equation from the perspective of computer vision. The research use pixel instead of point to describe the second framework which called Pixellary Pupil Distance [1] where pixellary introduces as a new word. The experi- ment of the Pixellary Pupil Distance proved that pixel equation can measure the unique Pupillary Distance for all human being.

Pixellary Pupil Distance is using pixel to measure unique distance because a pixel is the smallest unit of an image. It used RGB value. The red, green and blue use 8 bits each, which have integer values from 0 to 255 [2]. As A biometric information is an asset for every human [3] and The Euclidean Equation deals with two points which did not define any unique distance as a biometric information for pupillary distance [4]. Cartesian, Manhattan, vector projection equations are the most important equations to measure the distance between two points which did not give a unique distance of Pupils. After getting the failure result of the basic equation, This research investigated the proposed conceptual models and equations. This research has experimented a new biometric information as pupillary distance. This research is showing the experimentation of Pixellary Pupil Distance which calculated by the smallest unit of an image. This research also shows the Task description, dataset description, Comparison of Computation Time, Detection Rate, False Alarm Rate and Noise Reduction as Result.

The rest of the paper is organized as follows. The following section discusses literature review. Section 3 contains task description of the experiment. Section 4 discusses dataset. Section 5 discusses the result. Section 6 discusses Analysis and Discussion of the result. Section 7 discusses the Limitation and Future Work. Finally, This research concludes in section 8 .

\section{LITERATURE REVIEW}

The pupil is one of the smallest units of eyes. The pupil is located at the center of Irish. It was presented that there are two types of pupil according to its color [5]. They have studied several eye tracking systems such as E-learning, Irish recognition, Eye Control for Accessible and assistive, Car Assistant System and Field of View Estimation and they did not describe any experimental result.

Pupil localization is very important to detect pupil. It was widely investigated to identify pupil coordinate, center and centroid point using several algorithms. Pupil centroid and its coordinate have been 


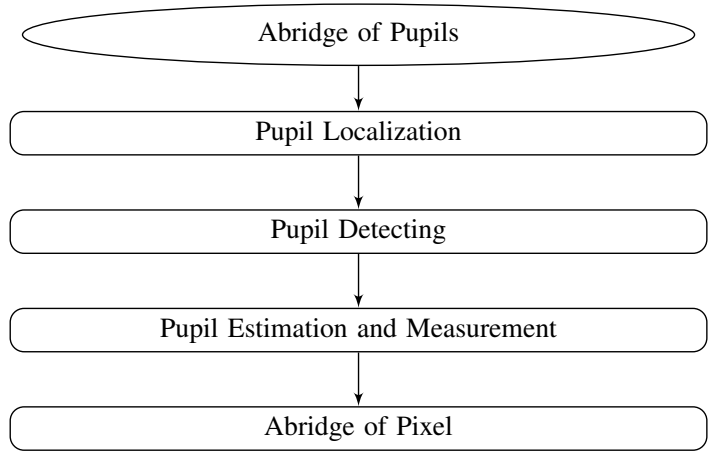

Fig. 1: A Block Diagram for Current Challenges of Pupils

used to localized pupil using Ada boosting eye classifier [6]. The Algorithm showed $47 \%$ accuracy for eye region detection in face region where the precision rate of detection in face region is $92 \%$. The accuracy of Ensembles Regression Tree [7] measured 85.75\% with $5 \%$ error rate and $99.7 \%$ accuracy recorded at $25 \%$ error rate after reinvestigation. On the other hand, Adaptive GBDT [8] recorded $99.89 \%$ accuracy at $25 \%$ error rate after reinvestigation where It localized pupil coordinate at $91.54 \%$ at $5 \%$ error rate. The Starburst model [9] has localized pupil center at the average frame rate of $196.76(\mathrm{~ms})$ where It recorded $91.92 \%$ accuracy.

Pupil detection is required to measure the accurate pupil distance using pixel value. The curvature algorithm [10] detect pupil center. The accuracy rate is $40 \%$ which is detected pupil boundary in an error of less 0.1 degrees. The detection of pupil sized is identified at $91 \%$ accuracy where $90 \%$ is the correct differentiation between narcoleptic and control subjects using The Modified Adaptive Resonance Theorem using NNs [11]. The Canny Algorithm [12] [13] able to detect pupil contour and edge at $10 \%$ error rate. Pupil contour is detected by Morphology algorithm [14] where the error rate is $10 \%$ on left pupil and $6 \%$ of right pupil. The Support Vector Machine(SVM) [15] is able to detect the coordinate of the area in eyes with approximately $1.73 \%$ error rate. On the other hand, Correlation matching and SVM are time consuming [15]. Although it had the highest accuracy.

The measurement and estimation are highly needed for accurate calculation of Pupil distance. It is the way to discover the pupil center and diameter and area. Pupil size and blink rate used to discover pupil boundary point by Dual Ellipse Fitting Method [16] in 2014. Novel self-tuning threshold method used for segmenting the pupil from background images where Blink detection accuracy is $99.7 \%$. The Eyelink system [7] estimated the gaze position and area is using the tracking of the eye data. The second order regressor of PA varied ranging from $0.02 \%$ to $95.4 \%$ in the left eye where it is $1.0 \%$ to $87.1 \%$ in the right eye. Transform [6], The Circle Area Equation [17] and Ellipse Equation [17] used to measure the diameter, center of the pupil in 2016. It was grated to use the Transform in measuring Pupil diameter and center point due to the accuracy level of Circle Area equation.

There are three major types of image which are Grayscale, Binary and RGB [18]. Pixel is the smallest unit of an image. Every pixel coincides with any one value. The value of the pixel between 0 and 255 in an 8-bit Grayscale image where zero is taken to be black, and 255 is taken to be white. The value of the pixel between 0 and 1 in a binary image where zero is taken to be black, and one is taken to be white. The value of the pixel between 0 and 255 in an RGB image.
RGB color space constructs all the colors from the combination of the Red, Green and Blue colors. The red, green and blue use 8 bits each, which have integer values from 0 to 255 [2]. The value of a pixel at any point corresponding to the intensity of the light photons striking at that point. Each pixel store a value proportional to the light intensity at that particular location. This research have used RGB color space to calculate the Pupillary Distance.

\section{TASK DESCRIPTION}

The measurement of Pupillary Distance experiment is the core task of this research. The experimental justification was the toughest challenge of this research. Although This research has experimented the system using MATLAB. This research has divided the experiment part into 7 sub-sections for describing convenience. Before This research is going to analyze the result of the experiment, it describes the experiment under section headline. The 7 sub-sections are:
(3.1) Face Detection
(3.2) Face Straighten
(3.3) Eye Detection
(3.4) Pupil Detection
(3.5) Pixel Extraction
(3.6) Pixel Distance Calculation
(3.7) Pupillary Distance Calculation

Besides that, This research describes dataset on Dataset Section. This research tested the proposed research and come up with pupillary distance which discusses at Result section. Then, Analysis and Discussion contains Comparison of Computation Time, Detection Rate, False Alarm Rate and Noise Reduction.

\subsection{Face Detection}

Face detection is the first step of purposed experiment. When proposed research get an image, it read the image. After reading the image, the proposed research will detect the face from the image. The Algorithm of face detection has been well established. SVM, Template Matching, Eigenvector, distributing based using Gaussian distribution and multilayer perceptron are the common and well performance algorithms [19]. The proposed research has divided the image into two halves for better detection. Proposed research used the existing algorithm of face detection with the help of Matlab. Cascade Object Detector is used to detect the face which is experimented on Viola-Jones algorithm [20].

\subsection{Face Straighten}

The SURF Operator is the local feature detector and descriptor. It has given invariance of scaling and rotation, strong robustness, and prominent separating capacity between different features [21]. Proposed research experiment face straightens for removing the initial noise. The Image which takes as input, those may not be center captured. Proposed research do face straighten for getting the accurate result.

\subsection{Eye Detection}

Eye detection is one of the major preprocessing stages before Irish and pupil detection said by Amer Al-Rahayfeh and Maid Faezipour [22]. Sobel filter can be used to extract edge feature. The proposed research found several Algorithm which used to detect eye using 
template matching [21]. Although, Support vector machine used to classify the images of an eye or non-eye patterns. As eyes are not symmetrical like as faces, one classifier of each eye used to train said by Yu Shiqi [20]. Cascade Object Detector is used to detect the eyes which are experimented according to Yu Shiqi algorithm.

\subsection{Pupil Detection}

Pupil detection is most important part of the paper. Several frameworks have been discussed pupil detection [19]. Those algorithms accuracy is not effective. As Proposed research need to pupil center and contour, its used Circle Equation Algorithm to detect the pupil. It has given low accuracy to detect pupil diameter [17]. The proposed research found that object polarity is dark. So, it modified the algorithm according to the sensitivity of darkness. Proposed research observed that pupil size is 6 pixel to 8 pixels. The proposed research found that the algorithm is better than exiting algorithm which is tested using Matlab.

\subsection{Pixel Extraction}

Pixel Extraction is one of the cores for features for distance calculation. The proposed research has found the pupil center co-ordinate and pupil size from pupil detection step. After getting the center coordinates of pupils, this research did average the value of $\mathrm{x}$-axis for removing pixel noise. Because $\mathrm{x}$-axis coordinate is not same. As a result, this research found same pupils size most of the cases which are reduced size and noise. Proposed research extracted the pupil contour using modified Circle Equation Algorithm. Proposed research also extracted the intermediate pixel of pupils which has set the value to zero.

\subsection{Pixel Distance Calculation}

The proposed research analyzes the only pixel of pupils and its intermediate. First, Proposed research calculates the no of rows. This research finds the first row of the left pupil of first non zero column and subtracts with the right pupil of first non zero column. This research also calculates a number of the pixel from the left pupil of first non zero column to right pupil of first non zero column in the same matrix. which this research called the pixel distance for a specific pixel of two pupils. This research carried the same calculation for all rows and column of left pupil to right pupil. when this research get any two zero column at the intermediate of a calculation, this research stop calculation for that row. because it's the finishing pixel of left pupil. This research also stores pixel co-ordinate of the right pupil for calculation.

In this step, this research has solved below equation [1]:

$$
P_{i}^{j}=L_{i}^{j}-R_{i}^{j}+\text { Distanceof } L_{i}^{j} \text { to } R_{i}^{j}
$$

\subsection{Pupillary Distance Calculation}

This research calculates work with the calculating result of Pixel Distance. This research sum the subtracted value of all specific pixel with its distance value. Then this research reorganizes the value as per subtracted value. Finally, this research sum all value which this research called pupillary distance.

In this step, this research experimented below equation [1]:

$$
\text { PupilDistance }=\sum_{i}^{j} P
$$

\section{DATASET}

This research used several databases. That dataset is not perfect for pupil detection. Some datasets used for face recognition. Specific datasets are used for Irish recognition. Since pupil is the smallest unit of an eye that's why those datasets are not given any significant result. For this reason, this research constructed a database with 20 peoples of multiple images. This research did not change the light effect. This research inspired the audience to make shocking eyes. So that this research can clearly capture the image of full Irish. As a result, the purposed framework detected the pupil from that image easily.

\section{RESULT}

This research has measured 20 subjects pupillary distance, each of 5 images. This research provides the information in the tabular format. This research shows the PD as pupillary distance, CT as computational time, FAR as False Alarming Rate and NRR as Noise Reduction Rate. The highest PD is 32432 and lowest is PD 18933 which is the average of 5 images without false recognition. The computational time shows an average time of 5 images where it defers from $1500 \mathrm{~ms}$ to $2100 \mathrm{~ms}$. As this research experiments $5 \mathrm{im}-$ ages of each subject which is $100 \%$ for a subject. False Alarming Rate is $20 \%$ when an image has been miscalculated of that subject, $40 \%$ means two images of that subject and so on. On the other hand, this research let $100 \%$ noise reduction if no false alarming has not occurred.

In Table 1, this research called 'subject' to one individual image, PD as pupillary distance, CT as Computational Time, FAR as False Alarming Rate and NRR as Noise Reduction Rate.

\section{ANALYSIS AND DISCUSSION}

The experimental result of the purposed framework has shown in tabular format in the immediate upper section where this research has shown the pupillary distance, computational time, False alarming rate and Noise Reduction. This research has shown the average pupillary distance of each individual. The lowest pupillary distance is 18933 and highest is 32432 . The average computational time of all subjects is $1813 \mathrm{~ms}$ where the lowest computational time is 1560 and highest is $2100 \mathrm{~ms}$. The False Alarming Rate occurred in $30 \%$ subjects. But the impact rate is $8 \%$ among all images. The noise reduction rate has been increased to $70 \%$ which is impact $92 \%$ images of the dataset which is shown in Fig-2.

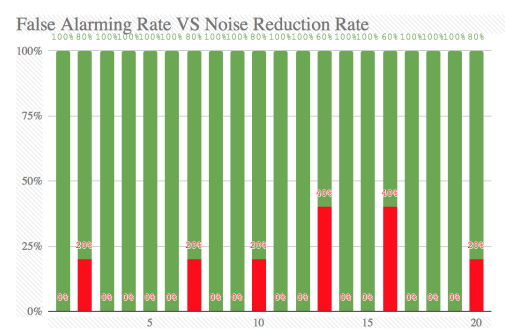

Fig. 2: The graph shows False Alarming Rate Vs Noise Reduction Rate

The lowest pupillary distance is 18933 at 9 th subject where computational time is $1680 \mathrm{~ms}$ which is lower than the average computational time of the dataset. The noise reduction rate is $100 \%$ where 
Table 1. : Result of 20 subjects each of 5 images

\begin{tabular}{|c|c|c|c|c|}
\hline Sub. No & PD & CT/Image & FAR & NRR \\
\hline 1 & 26983 & 1700 & $0 \%$ & $100 \%$ \\
\hline 2 & 20021 & 1600 & $20 \%$ & $80 \%$ \\
\hline 3 & 23002 & 1750 & $0 \%$ & $100 \%$ \\
\hline 4 & 25983 & 1850 & $0 \%$ & $100 \%$ \\
\hline 5 & 24029 & 1750 & $0 \%$ & $100 \%$ \\
\hline 6 & 23094 & 1800 & $20 \%$ & $100 \%$ \\
\hline 7 & 27128 & 2010 & $0 \%$ & $80 \%$ \\
\hline 8 & 30943 & 2050 & $0 \%$ & $100 \%$ \\
\hline 9 & 18933 & 1680 & $20 \%$ & $80 \%$ \\
\hline 10 & 32432 & 1930 & $0 \%$ & $100 \%$ \\
\hline 11 & 29123 & 1750 & $40 \%$ & $100 \%$ \\
\hline 12 & 22923 & 1980 & $0 \%$ & $60 \%$ \\
\hline 13 & 27452 & 1780 & $0 \%$ & $100 \%$ \\
\hline 14 & 21523 & 1920 & $40 \%$ & $100 \%$ \\
\hline 15 & 20329 & 1840 & $0 \%$ & $100 \%$ \\
\hline 16 & 19021 & 2100 & $0 \%$ & $100 \%$ \\
\hline 17 & 27392 & 1560 & $0 \%$ & $100 \%$ \\
\hline 18 & 23458 & 1660 & $20 \%$ & $80 \%$ \\
\hline 19 & 25123 & 1750 & & \\
\hline 20 & 23843 & 1800 & & $0 \%$ \\
\hline
\end{tabular}

the false alarming rate is $0 \%$. On the other hand, The highest pupillary distance is 32432 at 10th subject where computational time is $1930 \mathrm{~ms}$ which is greater than the average computational time of the dataset. The false alarming rate is $20 \%$ where the noise reduction rate is $80 \%$.

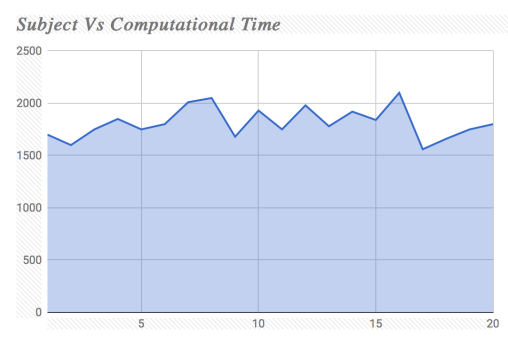

Fig. 3: The graph shows subject vs computational time

If this research looks the subject vs computational time graph, this research observed that computational time is in between $1560 \mathrm{~ms}$ to $2100 \mathrm{~ms}$, once a number of images are remaining same. The computational time is proportional to the pupillary distance where the False Alarming Rate or Noise Reduction Rate does not depend on pupillary distance. The pupillary distance is different of every individual at the same light intensity where it will be different for an individual if the light intensity change.

\section{LIMITATION AND FUTURE WORK}

This research has experimented pixellary pupil distance to calculate the pupillary distance which is given a unique result. This research has a scope to experiment with the biggest data set. On the other hand, this research did not provide any comparison with the millimeter scale. It is also a scope for further research improvement.This research did not distinguish between gender or age using the research output. This research did not consider with focal length, the light intensity which is biggest opportunity to research on pixellary pupil distance. Although, the proposed research will suggest you a new path to research on pupillary distance.

\section{CONCLUSION}

Pupillary distance is bio-metric information which is not proved by any researcher yet. But this research has shown a path to make it as biometric information. The challenge of pupil detection solved by the purposed framework and dataset. This research has also experimented pupillary distance according to the purposed framework which is the biggest milestone for computer vision. This research has shown that Modified Circle Algorithm can detect pupil better than all other algorithms to proposed framework. This research modified the object polarity, pupil size, and sensitivity to detect it more accurately. This research did not consider light intensity, focal length, age, gender. Finally, this research calculates the pupillary distance using pixel which has shown a straight path to the researcher to discover more about pupillary distance and there is the biggest scope to study about pupillary distance.

\section{REFERENCES}

[1] A. S. Saif, M. S. Hossain, and K. T. Hasan, "A descriptive conceptual framework to measure distance of pupil using pixel value," 6th International Conference on Informatics, Electronics , Vision, 2017.

[2] M. Popescu and R. T. Ionescu, Knowledge Transfer between Computer Vision and Text Mining:: Similarity-based Learning Approaches. Springer, 2016.

[3] R. Bhatia, "Biometrics and face recognition techniques," International Journals of Advanced Research in Computer Science and Software Engineering (IJARCSSE), vol. 3, no. 2, 2013.

[4] S. Ganga, "Chapter 4- methods for measuring distance in images." 
[5] V. Roselin.E.Chirchi, Dr.L.M.Waghmare, and E.R.Chirchi, "Iris biometric recognition for person identification in security systems," International Journal of Computer Applications, pp. 0975 - 8887, 2011.

[6] G. Zhang, J. Chen, G. Su, and J. Liu, "Double-pupil location of face images," Pattern Recognition, vol. 46, pp. 642-648, 2013.

[7] K. W. Choe, R. Blake, and S.-H. Lee, "Pupil size dynamics during fixation impact the accuracy and precision of videobased gaze estimation," Vision Research, 2015.

[8] D. Tian, G. He, J. Wu, H. Chen, and Y. Jiang, "An accurate eye pupil localization approach based on adaptive gradient boosting decision tree," in Visual Communications and Image Processing (VCIP). IEEE, 2016.

[9] Y. Zhao, Z. Qu, H. Han, and L. Yuan, "An effective and rapid localization algorithm of pupil center based on starburst model," in Advanced Information Management, Communicates, Electronic and Automation Control Conference (IMCEC). IEEE, 2016.

[10] D. Zhu, S. T. Moore, and T. Raphan, "Robust pupil center detection using a curvature algorithm," Computer Methods and Programs in Biomedicine, vol. 59, pp. 145-157, 1999.

[11] D. Liu, Z. Pang, and S. R. Lloyd, "A neural network method for detection of obstructive sleep apnea and narcolepsy based on pupil size and eeg," IEEE TRANSACTIONS ON NEURAL NETWORKS, vol. 19, no. 2, pp. 308-318, 2008.

[12] G. Xin, C. Ke, and H. Xiaoguang, "An improved canny edge detection algorithm for color image," in Industrial Informatics (INDIN), 2012 10th IEEE International Conference on, 2012.

[13] C. Jayachandra and H. Reddy, "Iris recognition based on pupil using canny edge detection and kmeans algorithm," in International Journal Of Engineering And Computer Science, vol. 2, no. 1, 2013, pp. 221-225.

[14] A. B. Roiga, M. Moralesb, J. Espinosaa, J. Pereza, D. Masa, and C. Illueca, "Pupil detection and tracking for analysis of fixational eye micromovements," Optik, vol. 123, pp. 11-15, 2012.

[15] C. Yan, Y. Wang, and Z. Zhang, "Robust real-time multi-user pupil detection and tracking under various illumination and large-scale head motion," Computer Vision and Image Understanding, vol. 115, pp. 1223-1238, 2011.

[16] S. Chen and J. Epps, "Efficient and robust pupil size and blink estimation from near-field video sequences for humanmachine interaction," IEEE Transactions on Cybernetics, vol. 44, no. 12, pp. 2356-2367, 2014.

[17] J. N. Sari, H. A. N, L. E. N, P. I. Santosa, and R. Ferdiana, "A study on algorithms of pupil diameter measurement," in Science and Technology-Computer (ICST), International Conference on, 2016.

[18] S. Birchfield, Image Processing and Analysis. Cengage Learning, 2016.

[19] A. S. Saif and M. S. Hossain, "A study of pupil orientation and detection of pupil using circle algorithm::a review," International Journal of Engineering Trends and Technology (IJETT), vol. 54, no. 1, 2017.

[20] Mathworks, "vision.cascadeobjectdetector system object."

[21] M.-H. Yang, D. J. Kriegman, and N. Ahuja, "Detecting faces in images: A survey," IEEE Transactions on Pattern Analysis and Machine Intelligence, vol. 24, no. 1, pp. 34-58, 2002.
[22] A. Al-Rahayfeh and M. Faezipour, "Eye tracking and head movement detection: A state-of-art survey," IEEE Journal of Translational Engineering in Health and Medicine, 2013. 